\title{
Exposición ocupacional a radiaciones ultravioleta UVA/UVB de los trabajadores agrícolas de la provincia de Cartago, Costa Rica
}

\section{Occupational exposure to ultraviolet radiation (UVA UVB) in agricultural workers in Cartago, Costa Rica}

\author{
Tannia Araya-Solano ${ }^{1}$, Esteban Arias-Monge ${ }^{2}$, \\ Andres Robles-Ramírez ${ }^{3}$, Ara Villalobos-Rodríguez ${ }^{4}$
}

Fecha de recepción: 29 de setiembre de 2019

Fecha de aprobación: 3 de febrero de 2020

Araya Solano, T; Arias Monge, E; Robles Ramirez, A; Villalobos Rodriguez, A. Exposición ocupacional a radiaciones ultravioleta UVA/UVB de los trabajadores agrícolas de la provincia de Cartago, Costa Rica. Tecnología en Marcha. Vol. 34-1. Enero-Marzo 2021. Pág 104-114.

doi) https://doi.org/10.18845/tm.v34i1.4486

1 M.Sc. Salud Ocupacional. Escuela de Ingeniería en Seguridad Laboral e Higiene Ambiental, Instituto Tecnológico de Costa Rica, Cartago. Costa Rica. Correo electrónico: taraya@tec.ac.cr (D) https://orcid.org/0000-0001-9465-1530

2 M.Sc. Salud Ocupacional. Escuela de Ingeniería en Seguridad Laboral e Higiene Ambiental, Instituto Tecnológico de Costa Rica, Cartago. Costa Rica. Correo electrónico: esarias@tec.ac.cr (D) https://orcid.org/0000-0001-9728-5310

3 Mtr. en Sistemas de Manufactura. Escuela de Ingeniería en Seguridad Laboral e Higiene Ambiental, Instituto Tecnológico de Costa Rica, Cartago. Costa Rica. Correo electrónico: anrobles@tec.ac.cr (iD https://orcid.org/0000-0002-5227-5685

4 Mtr. en Gestión de Proyectos. Escuela de Ingeniería en Seguridad Laboral e Higiene Ambiental, Instituto Tecnológico de Costa Rica, Cartago. Costa Rica. Correo electrónico: avillalobos@tec.ac.cr 


\title{
Palabras clave
}

Radiación ultravioleta; exposición solar; trabajadores agrícolas.

\section{Resumen}

La radiación ultravioleta constituye una fuente de riesgo para el sector agrícola; en Costa Rica no se han publicado datos sobre la exposición a este agente físico. Por tal motivo, se desarrolló una investigación exploratoria con dos asociaciones de agricultores ubicadas en la zona norte y El Guarco, en la provincia de Cartago, cuyo objetivo fue el de cuantificar el nivel de radiación ultravioleta al que se exponen los colaboradores de las zonas agrícolas, así como estimar la dosis eritema estándar y establecer una matriz para determinar el nivel de riesgo por exposición a la radiación solar. Las mediciones iniciaron en noviembre de 2015 y finalizaron en enero de 2017, en horario de 6:00 hasta las 15:00 horas aproximadamente, iniciando en noviembre de 2015 y finalizando en enero de 2017 ; se aplicaron métodos estadísticos como promedios ponderados, máximos, mínimos y desviación estándar de los datos obtenidos y una encuesta a los trabajadores para determinar el fototipo de piel y las zonas de esta más expuestas.

Los principales resultados mostraron que la irradiación mínima reportada fue de 1,9 W/cm² y la máxima, de 160,9 W/cm²; los niveles promedio más altos se reportaron entre las 10:00 y las 13:00 horas. La época del año con mayor nivel de irradiación fue la seca, en la zona alta, con $167 \mathrm{~W} / \mathrm{cm}^{2}$ como máximo. La dosis eritema estándar estuvo entre 2,3 SED y 9,8 SED, que sobrepasa lo recomendado por la Comisión Internacional de lluminación (1,09 SED). El índice de radiación ultravioleta se encontró entre 8 y 12, clasificado como alto y muy alto. Bajo las condiciones descritas podría aumentar el riesgo para los colaboradores de las fincas agrícolas, de eritemas en la piel. Se planteó la matriz de riesgo por exposición, tomando en cuenta aspectos como tarea o puesto, altitud, época del año, nivel de irradiación, parte del cuerpo expuesta y fototipo de piel.

\section{Keywords}

Ultraviolet radiation; solar exposure; agricultural workers.

\begin{abstract}
Ultraviolet radiation constitutes a source of risk for the agricultural sector. In Costa Rica, no data have been published on the exposure to this physical agent, for this reason an exploratory research has been developed whose objective was to quantify the level of ultraviolet radiation to which the collaborators of the agricultural areas (north zone and El Guarco, in Cartago) are exposed, as well as to estimate the standard erythema doce. The radiation level was measured from 6:00 until approximately 15:00 hour. The data were analyzed using statistical methods, and farm workers were surveyed to determine the skin phenotype, most exposed areas of the skin, and other information. The main results showed that the minimum irradiance reported was $1,9 \mathrm{~W} / \mathrm{cm}^{2}$, and the maximum was $160,9 \mathrm{~W} / \mathrm{cm}^{2}$; the highest average levels were reported between 10:00 and 13:00 hours. The time of the year with the highest level of irradiance was the dry season, in the north zone, with $167 \mathrm{~W} / \mathrm{cm}^{2}$ as maximum. The standard erythema dose was estimated between 2,3 and 9,8, exceeding that recommended by the International Commission on Illumination. The ultraviolet index was found between 8 and 12, classified as high and very high. Under the conditions described, the risk of erythema for the collaborators in the farms was increased. A matrix is proposed to determine risk by exposure, taking into account aspects such as task or position, altitude, time of year, level of irradiance, part of the body exposed, and skin phenotype.
\end{abstract}




\section{Introducción}

Las radiaciones corresponden a una forma de energía con un amplio espectro electromagnético [1], que contiene luz ultravioleta, luz visible y la radiación infrarroja, entre otras longitudes de onda [2]. Específicamente, la radiación ultravioleta comprende a una parte de la energía radiante que proviene del sol [3], que tiene longitudes de onda más cortas que la luz visible, pero más largas que los rayos X [4], en el rango de 200 a 400 nanómetros [5], y se distribuye como luz ultravioleta $A, B$ y $C$. Las radiaciones $A$ y $B$ son las que inciden en la superficie de la Tierra y, por lo tanto, en nuestra piel [6], debido a que la $\mathrm{C}$ es filtrada en su totalidad por la capa de ozono [7].

Si bien la capa de ozono actúa como un escudo que protege a los organismos y ecosistemas de las consecuencias que se podrían generar producto de la radiación ultravioleta [8], la disminución de ella ha dado lugar a la necesidad de comunicar a la población sobre los posibles efectos que se pueden presentar en las personas [9].

Los efectos en la salud por la radiación ultravioleta principalmente se manifiestan en daños en la piel [10]. Según Zuluaga [2], pueden ser agudos o crónicos; el daño agudo es el que se presenta como una quemadura solar y el daño crónico, el que produce fotoenvejecimiento y formación de cataratas corticales [11]. Entre otras de las lesiones por la exposición a radiación ultravioleta se encuentra el cáncer de piel [12] [13], el cual ha tenido un considerable aumento en su incidencia [14], principalmente por la exposición prolongada a rayos B, debido a que estos penetran en la piel afectando la epidermis y, por ende, generando daño en el ADN [6], así como a rayos $A$, los que aumentan el número de células inflamatorias dérmicas [15].

En nuestro país, el cáncer de piel es el tipo más frecuente. Para el 2016, la tasa de incidencia de tumores en piel era de 58,04\%, en hombres provenientes de la Gran Área Metropolitana [16]. En países como los Estados Unidos, ocurren aproximadamente un millón de nuevos casos de este tipo de cáncer anualmente [17], debido a conductas inadecuadas como la exposición solar desde edades muy tempranas, o el aplicarse el bloqueador solar en una sola ocasión al día, entre otras [18].

Uno de los principales factores de riesgo relacionado con muchos tipos de cáncer corresponde a las condiciones ambientales [19]. Específicamente en el riesgo de cáncer de piel, los niveles de radiación pueden ser influenciados por la hora del día [20], la localización geográfica o los elementos de la topografía [13] como montañas, lagos o valles, los cuales inciden en la cantidad de radiación que recibe un lugar [21]. Las condiciones climáticas, específicamente las nubes, por otra parte, pueden tener un efecto positivo en la reducción de gran parte de la energía solar [22], especialmente la infrarroja, llegando a evitar el paso de hasta un 50\% o más de la UVB [11].

El aumento de las lesiones en la piel producto de la radiación ultravioleta también se asocia a intensidad [23] y su distribución sobre el cuerpo [24], y afecta principalmente a las personas que trabajan durante largas horas, expuestas a las radiaciones ultravioleta [25], especialmente en labores que se realizan al aire libre [26], como los trabajos agrícolas [27].

De acuerdo a Gies, Glanz, O'Riordan et al.[28]los trabajadores al aire libre son grupos que reciben radiación solar ultravioleta en forma regular y en dosis significativas, que con frecuencia sobrepasan los valores recomendados [28]. Frente a este hecho, factores como el índice ultravioleta (IUV), el cual es una medida de la radiación ultravioleta proveniente del sol en la superficie de la Tierra y es un indicador cuantitativo del efecto de este tipo de radiación sobre la piel humana, permiten generar conciencia en la población trabajadora sobre la importancia de tomar medidas de protección ante la exposición a la radiación UV [29]. Además, valores como la dosis eritema estándar (SED), que establece un límite de dosis recomendado (TLV) de 
109J/m² (equivalente a 1,09 SED) para un periodo de 8 horas [30], tienen como fin proteger a los trabajadores de los posibles efectos de la radiación ultravioleta en la salud [31].

Consecuente con lo anterior, el proyecto que aquí se resume tuvo como objetivo cuantificar el nivel de radiación (UVA/UVB) y conocer las variables que pueden tener mayor incidencia en el riesgo, para generar un modelo de exposición que sirva de insumo en la toma de decisiones de alcance nacional por parte de las diferentes entidades de Salud. El proyecto obedece a la urgencia de programas de protección contra la radiación solar que impulsen cambios en los estilos de vida, según lo indica la Organización Mundial de la Salud [30], para frenar la tendencia al aumento de los casos de cáncer de piel.

\section{Metodología}

La investigación se realizó en la provincia de Cartago, específicamente en Tierra Blanca ( $9^{\circ} 55^{\prime}$ $\mathrm{N}, 83^{\circ} 53^{\prime} \mathrm{W}$, con una altitud mayor de $2080 \mathrm{~m}$ sobre el nivel del mar) y Tejar del cantón de El Guarco (9 $50^{\prime} \mathrm{N}, 83^{\circ} 59^{\prime} \mathrm{W}$, con una altitud de $1440 \mathrm{~m}$ sobre el nivel del mar) en la época Iluviosa y la época seca.

En el estudio participaron los trabajadores de fincas pertenecientes a la Asociación de Horticultores del Irazú (AHSORI) y la Asociación de Agricultores de El Guarco (AGRIRETEC). El estudio incluyó un total de 16 fincas; la muestra, seleccionada de las fincas, fue determinada a conveniencia, para un total de 34 adultos, todos hombres.

Encuesta higiénica: Al inicio de la investigación se visitó cada una de las fincas dispuestas a participar, con el fin de aplicar la encuesta higiénica, para luego plantear la estrategia de muestreo. Entre los aspectos que se tomaron en cuenta figuran jornada laboral, tipo de cultivo y lugar exacto de la finca.

De acuerdo a Sierra [32], el incidente ponderado de eritema en sitios anatómicos durante un período específico de tiempo se conoce como exposición a la radiación ultravioleta (RUV).

Irradiación: se midió con un radiómetro (Sper Scientific UVA/B Light Meter, Modelo 850009), que es un medidor de la luz ultravioleta (UV), para espacios naturales y artificiales. El radiómetro se colocó en un trípode, en posición sur (siempre con el uso de coordenadas geográficas) respecto a la posición del sol. El equipo registró datos cada 10 min durante toda la jornada.

Niveles de irradiación: Se calcularon promedios, máximos, mínimos y desviación estándar para los datos obtenidos por finca, así como los obtenidos por estación lluviosa y seca, y ubicación en zona alta y zona baja.

Dosis eritema estándar (SED) del estudio $\left(\mathrm{J} / \mathrm{m}^{2}\right)$ : Se determinó el fototipo de los participantes según la clasificación de tipos de piel de Fitzpatrick [32], con base en una entrevista aplicada a cada trabajador sobre información personal (i.e., tipo de piel, color de cabello y ojos, tendencia al bronceado, áreas del cuerpo expuestas al sol, consideraciones sobre la vestimenta, hábitos de protección solar, tiempos de descanso, historial de trabajo e información familiar sobre el cáncer de piel).

Para proceder con el análisis de la RUV con relación a las actividades realizadas, se efectuaron observaciones, sin interacción con los participantes, considerando la postura asumida por el trabajador, los sitios anatómicos expuestos al sol y el equipo de protección personal utilizado. Se asignó un peso específico a cada una de las subcategorías indicando la medida de la exposición directa a la RUV según las actividades del ciclo de producción. La RUV diaria en cada finca, según los valores obtenidos de irradiación incidente en toda la jornada laboral, se describe por medio de las unidades SED. Se requiere, según Sierra [32], una exposición de 
aproximadamente 1,5 a 3,0 SED para producir un eritema perceptible en la piel no aclimatada blanca, sin protección.

La estimación de SED en cada finca se calculó según la irradiación medida y los valores máximos obtenidos, tomando en consideración el periodo de las 11:00 a las 13:00 horas.

También se reportaron los datos del índice UV, según el Instituto Meteorológico Nacional (IMN).

Matriz para estimar el riesgo por exposición a la radiación solar: Con la información obtenida de los valores de irradiación, características de la tarea (postura, vestimenta), fototipo de piel y demás variables, se procedió a la confección de una matriz que permitió establecer el nivel de riesgo, y no solo el nivel de irradiación, mediante la definición de la ecuación planteada.

Para efectos del estudio, las fincas se nombraron con letras mayúsculas iniciando en $A$ terminando $P$.

\section{Resultados y discusión}

\section{Datos generales}

Los productores participantes en el proyecto se ubicaban en la zona norte y Tejar de El Guarco, de Cartago. De la primera ubicación participaron 14 fincas y de la segunda, 2 fincas, para un total de 16 fincas. En el momento de la toma de datos, las actividades realizadas eran la preparación del terreno, la siembra, la cosecha y el atomizado, de productos como zanahoria, cebolla y culantro. Todas las tareas se realizaban al aire libre. En la mayoría de los casos, los tiempos de comida igualmente se tomaban al aire libre; en algunos otros, se contaba con carpas o una bodega.

La jornada laboral comprendía entre 8 y 9 horas laborales, dependiendo de las tareas que se tuvieran que realizar.

Las fincas F y L se ubicaban en Tejar de El Guarco, y el resto en la zona norte de la provincia. La ubicación de las fincas se muestra en la figura 1.

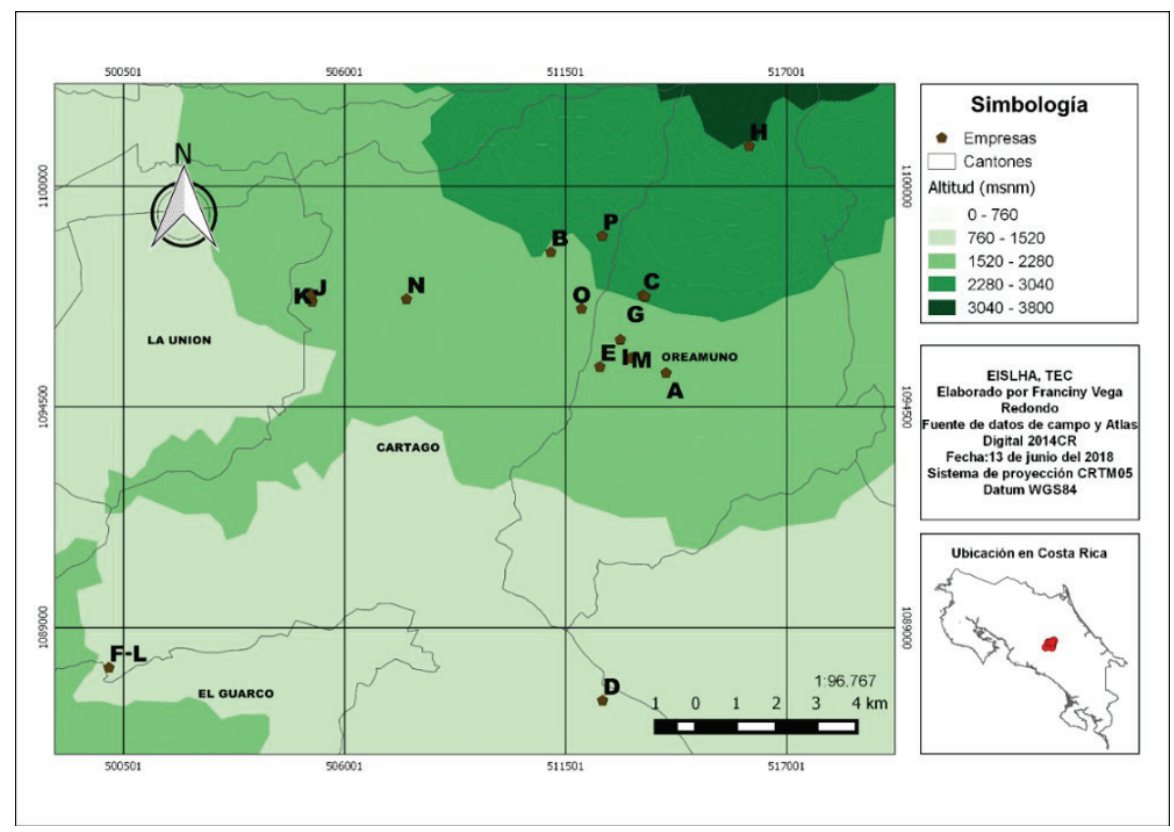

Figura 1. Ubicación de las fincas incluidas en el estudio. 


\section{Niveles de irradiación UVA/UVB}

Las mediciones de irradiación se realizaron entre las 7:00 y las 15:00 horas. En el cuadro 1 se muestran los promedios, la desviación estándar, los máximos y mínimos de los niveles de irradiación obtenidos por cada finca, así como datos generales de ellas.

Cuadro 1. Niveles de irradiación promedio, desviación estándar, máximo y mínimo medidos en W/m² para cada una de las fincas, así como ubicación, altitud, cantidad de trabajadores y tipo de cultivo.

\begin{tabular}{|c|c|c|c|c|c|c|c|c|}
\hline $\begin{array}{l}\mathrm{N}^{\circ} \\
\text { Finca }\end{array}$ & $\begin{array}{c}\operatorname{Min}(\mathrm{W} / \\
\left.\mathrm{cm}^{2}\right)\end{array}$ & $\begin{array}{c}\text { Prom (W/ } \\
\left.\mathrm{cm}^{2}\right)\end{array}$ & $\begin{array}{c}\operatorname{Max}(\mathrm{W} / \\
\left.\mathrm{cm}^{2}\right)\end{array}$ & $\mathrm{DE}$ & $\begin{array}{l}\text { Altitud } \\
\text { (m.s.n.m) }\end{array}$ & Latitud & Longitud & $\begin{array}{c}\text { Cant. } \\
\text { trabajadores } \\
\text { (población) }\end{array}$ \\
\hline A & 16,5 & 52,8 & 95,9 & 19,9 & 1989 & $9^{\circ} 54^{\prime} 21{ }^{\prime \prime} \mathrm{N}$ & $83^{\circ} 52^{\prime} 20^{\prime \prime} 0$ & 20 \\
\hline B & 23,9 & 96,3 & 151,9 & 32,3 & 2181 & $9^{\circ} 55^{\prime} 59^{\prime \prime} \mathrm{N}$ & $83^{\circ} 53^{\prime} 54^{\prime \prime} 0$ & 15 \\
\hline$C$ & 30,1 & 85,5 & 153,7 & 32,2 & 2281 & $9^{\circ} 55^{\prime} 24 " \mathrm{~N}$ & $83^{\circ} 52^{\prime} 39^{\prime \prime} 0$ & 5 \\
\hline $\mathrm{D}$ & 18,4 & 70,4 & 139,9 & 29,9 & 2305 & $9^{\circ} 55^{\prime} 53^{\prime \prime} \mathrm{N}$ & $83^{\circ} 53^{\prime} 15^{\prime \prime} \mathrm{O}$ & 7 \\
\hline $\mathrm{E}$ & 3,1 & 45,7 & 85,1 & 33,8 & 2030 & $9^{\circ} 54^{\prime} 26 " \mathrm{~N}$ & $83^{\circ} 53^{\prime} 14^{\prime \prime} 0$ & 18 \\
\hline $\mathrm{F}$ & 1,9 & 48,2 & 101,1 & 27,6 & 1476 & $9^{\circ} 50^{\prime} 22 " \mathrm{~N}$ & $83^{\circ} 59^{\prime} 55^{\prime \prime}$ & 10 \\
\hline G & 33,2 & 110,6 & 167 & 38,6 & 2262 & $9^{\circ} 55^{\prime} 23^{\prime \prime} \mathrm{N}$ & $83^{\circ} 52^{\prime} 37^{\prime \prime} \mathrm{O}$ & 6 \\
\hline $\mathrm{H}$ & 29,3 & 111,6 & 160,9 & 38,1 & 3062 & $9^{\circ} 57^{\prime} 25^{\prime \prime} \mathrm{N}$ & $83^{\circ} 51^{\prime} 12^{\prime \prime} \mathrm{O}$ & 6 \\
\hline 1 & 37,1 & 111,5 & 142,6 & 35,4 & 2118 & $9^{\circ} 54^{\prime} 48^{\prime \prime} \mathrm{N}$ & $83^{\circ} 52^{\prime} 57^{\prime \prime}$ & 12 \\
\hline J & 27,6 & 93,3 & 147,4 & 40,7 & 2090 & $9^{\circ} 55^{\prime} 19^{\prime \prime} \mathrm{N}$ & $83^{\circ} 57^{\prime} 09^{\prime \prime} \mathrm{O}$ & 26 \\
\hline K & 24,3 & 95,0 & 134,7 & 33,9 & 2332 & $9^{\circ} 55^{\prime} 25^{\prime \prime} \mathrm{N}$ & $83^{\circ} 57^{\prime} 09^{\prime \prime}$ & 15 \\
\hline L & 24,3 & 86,7 & 119,4 & 26,9 & 1473 & $9^{\circ} 50^{\prime} 22 " \mathrm{~N}$ & $83^{\circ} 59^{\prime} 55^{\prime \prime}$ & 9 \\
\hline M & 24,2 & 87,9 & 129 & 32,6 & 2124 & $9^{\circ} 54^{\prime} 33 \mathrm{~N}$ & $83^{\circ} 52^{\prime} 49^{\prime \prime} \mathrm{O}$ & 12 \\
\hline $\mathrm{N}$ & 22,1 & 45,4 & 90 & 29,6 & 1938 & $9^{\circ} 55^{\prime} 21 \mathrm{~N}$ & $83^{\circ} 55^{\prime} 52^{\prime \prime} 0$ & 8 \\
\hline 0 & 25 & 81,2 & 137,9 & 35,7 & 2219 & $9^{\circ} 55^{\prime} 13 \mathrm{~N}$ & $83^{\circ} 53^{\prime} 29^{\prime \prime}$ & 7 \\
\hline$P$ & 20,7 & 79,3 & 107,1 & 28,9 & 2369 & $9^{\circ} 56^{\prime} 12 \mathrm{~N}$ & $83^{\circ} 53^{\prime} 12^{\prime \prime} 0$ & 6 \\
\hline
\end{tabular}

De acuerdo con la información anterior, el promedio más alto se registró en la zona norte en condiciones soleadas y el más bajo, en Tejar, en un día nublado.

El nivel de irradiación es muy variable, por condiciones como la nubosidad presentada en el momento de realizar la medición, independientemente de la zona (alta o baja), por lo que no se pudo establecer una tendencia.

Se calculó el promedio, el máximo, el mínimo y la desviación estándar de los datos agrupados por franja horaria, ubicación (zona alta o baja) y época del año (ver cuadro 2).

Según la agrupación por franja horaria, en promedio entre las 13:00 y las 15:00 horas la irradiación fue mayor; sin embargo, el valor máximo se dio entre las 10:00 y las 12:59 horas. Este valor coincide con el reportado como máximo en la zona norte de Cartago y en época seca. Los datos de irradiación se pudieron ver afectados por la nubosidad que se presentó tanto en época seca como lluviosa. 
Cuadro 2. Promedios, mínimos y máximos de la irradiación, en diferentes condiciones.

\begin{tabular}{|c|c|c|c|c|}
\hline Condición & Promedio $\left(\mathrm{W} / \mathrm{cm}^{2}\right)$ & Máximo $\left(\mathrm{W} / \mathrm{cm}^{2}\right)$ & $\begin{array}{c}\text { Mínimo }(\mathrm{W} / \\
\left.\mathrm{cm}^{2}\right)\end{array}$ & Desv. est \\
\cline { 1 - 4 } Franja horaria & 57,6 & 127,0 & 0,3 & 26,8 \\
\hline 6:00-9:59 & 94,9 & 167,0 & 3,1 & 36,4 \\
\hline 10:00-12:59 & 95,8 & 148,5 & 8,6 & 34,8 \\
\hline 13:00-15:00 & 83,9 & 167,0 & 0,3 & 37,8 \\
\hline $\begin{array}{c}\text { Zona alta (zona norte) } \\
\text { Zona baja (Tejar de El } \\
\text { Guarco) }\end{array}$ & 67,4 & 119,4 & 1,9 & 33,4 \\
\hline Seca & 90,5 & 167,0 & 0,30 & 38,3 \\
\hline Lluviosa & 72,1 & 153,7 & 1,90 & 34,4 \\
\hline
\end{tabular}

\section{Dosis eritema estándar (SED) en los trabajadores agrícolas}

Con base en los resultados de la encuesta aplicada, se determinó que la postura ergonómica según la actividad realizada era de pie o inclinada; la vestimenta utilizada por los colaboradores era pantalón de mezclilla y camisa de botones y manga larga.

Las partes del cuerpo con mayor exposición al sol, para el total de los colaboradores, eran cara $(100 \%)$, pecho $(100 \%)$ y manos $(50 \%)$.

Con respecto al tipo de piel, según la clasificación Fitzpatrick, se encontró que el fototipo predominante fue el IV, seguido del III, lo que significa una sensibilidad normal y tolerancia a la luz solar en la piel. En la figura 2 se muestra la información.

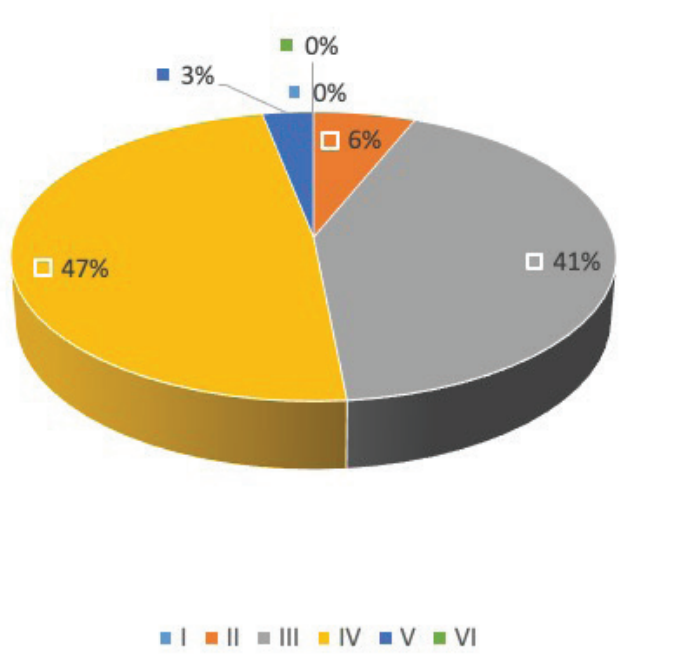

Figura 2. Fototipo de piel de los colaboradores. Fuente: M. Sierra, 2016

En el cuadro 3 se muestra el resultado del SED por cada finca. 
Cuadro 3. Niveles máximos de radiación, SED, en cada una de las fincas.

\begin{tabular}{|c|c|c|c|}
\hline N Finca & Max $\left(W / \mathrm{m}^{2}\right)$ & SED & $\begin{array}{c}\text { Índice UV } \\
\text { reportado por } \\
\text { IMN }\end{array}$ \\
\hline A & 95,9 & 4,1 & 9 \\
\hline B & 151,9 & 7,4 & 9 \\
\hline C & 153,7 & 6,0 & 9 \\
\hline D & 139,9 & 6,0 & 9 \\
\hline E & 85,1 & 3,9 & 8 \\
\hline F & 101,1 & 2,3 & 10 \\
\hline G & 167 & 9,4 & 8 \\
\hline H & 160,9 & 9,8 & 10 \\
\hline I & 142,6 & 9,8 & 11 \\
\hline J & 147,4 & 9,1 & 12 \\
\hline K & 134,7 & 8,5 & 12 \\
\hline L & 119,4 & 7,2 & 12 \\
\hline M & 129 & 7,4 & 10 \\
\hline
\end{tabular}

Fuente: M. Sierra, 2016

La CIE (2001) indica que, si la exposición está entre 1,5 y 3,0 SED, se puede producir un eritema perceptible en la piel no aclimatada blanca sin protección [33]. En el estudio todos los individuos fueron aclimatados, pero los valores de SED resultaron mayores a los recomendados, por lo que se encontraban en riesgo.

De acuerdo a los datos obtenidos por el IMN, se registraron valores del índice UV categorizados como muy altos o extremos [34], lo que podía aumentar el riesgo de daños en la piel, especialmente en áreas como la cara, que como se mencionó anteriormente, fue una de las partes con mayor exposición al sol. Además, se encontró que, aunque los trabajadores utilizaban gorras o sombreros, la práctica de aplicación del bloqueador solar era realizada solamente una vez al día.

Se determinó que las horas más críticas fueron entre las 10:00 y las 13:00 horas en días de verano; después de las 13:00 horas el nivel decaía. Igualmente, en la época seca se encontró el nivel más alto de irradiación. Según Narváez (2001), las nubes disminuyen la recepción de energía solar, y pueden evitar el paso de 50\% o más de la UVB; este hecho se comprobó en las fincas $B, C, D, E, F, G, H, N, O, Q$ y $P$, principalmente, donde se encontraron descensos en los niveles de radiación.

\section{Matriz para estimación de la RUV}

Partiendo de los resultados expuestos, se elaboró la matriz para la determinación de la exposición a la radiación solar, la cual contempla la altitud de la zona geográfica en la cual se está desarrollando la tarea, la época del año en la cual se están tomando los datos de irradiación, la parte del cuerpo expuesta y el fototipo de piel. Estos valores se ponderan según el criterio de los investigadores y en función de los resultados del análisis de las variables, para proceder a obtener un nivel de riesgo por tarea específica evaluada, de manera tal que de dicha matriz se puede también obtener un nivel máximo de exposición y determinar cuáles variables poseen mayor peso en una evaluación a futuro. 
Matriz para la estimación de la exposición a radiación solar

\begin{tabular}{|c|c|c|c|c|c|c|c|c|c|c|c|c|c|}
\hline \multirow{3}{*}{ Tarea o puesto } & \multirow{2}{*}{\multicolumn{2}{|c|}{ Altitud }} & \multicolumn{2}{|c|}{ Época del año } & \multirow{2}{*}{\multicolumn{2}{|c|}{$\begin{array}{c}\text { Nivel de alarma de } \\
\text { irradiancia }\end{array}$}} & \multirow{2}{*}{\multicolumn{3}{|c|}{ Parte del cuerpo expuesta }} & \multirow{2}{*}{\multicolumn{3}{|c|}{ Fenotipode piel }} & \multirow{3}{*}{ NR } \\
\hline & & & \multirow{2}{*}{ Verano } & \multirow{2}{*}{ Invierno } & & & & & & & & & \\
\hline & $1400 \mathrm{msnm} \leq>1900 \mathrm{msnm}$ & 1950 msnm $\geq$ & & & $\leq 60$ & $60 \leq$ & Cuello & Cara & Manos & Tipo 1-2 & Tipo3-4 & Tipo5-6 & \\
\hline \multicolumn{14}{|l|}{ Recoleeción } \\
\hline \multicolumn{14}{|l|}{ Siembra } \\
\hline & & & & & & & & & & & & & \\
\hline & & & & & & & & & & & & & \\
\hline & & & & & & & & & & & & & \\
\hline
\end{tabular}

Figura 3. Matriz para la estimación de la RUV.

La matriz de la figura 3 parte de una ponderación propuesta por los investigadores que atiende a la ecuación (NR) y que tiene el potencial de ser aplicada y realimentada mediante su uso en evaluaciones de campo y futuras investigaciones.

$$
N R=\text { altitud }+ \text { época }+ \text { nivel de alarma }+ \text { partes del cuerpo expuestas }+ \text { fototipo de piel }
$$

En donde

$N R=$ nivel de riesgo

Altitud $=$ valor asignado según el rango de medición

Época del año = valor asignado según la estación (2 para verano;1 para invierno)

Nivel de alarma $=$ valor asignado de 2 al superar el valor de alarma

Partes del cuerpo expuestas $=1$ por cada parte observada con exposición

Fototipo de piel = valor asignado según el fototipo (1-2:2);(3-4:1);(5-6:0)

\section{Conclusiones y recomendaciones}

Los niveles de irradiación aumentan conforme transcurre el día, siendo las horas más críticas entre las 12:00 y las 13:00, por lo que existe un factor de riesgo relacionado con la hora en la que se encuentre laborando el trabajador.

La dosis eritema sobrepasa lo recomendado por la ICNIRP, (International Commission On Nonlozing Radiation Protection) [31], por lo que hay probabilidad de que se presenten eritemas en los colaboradores; en cara, pecho y manos, que son las partes más expuestas de la piel.

Los resultados obtenidos no son los esperados debido a que algunas de las variables analizadas quedaron fuera del control tanto de las fincas participantes como de los investigadores, por ejemplo, las características de la vestimenta y algunos aspectos propios de posturas al realizar 
la tarea; por tal motivo no se pudo realizar un modelo que permitiera efectuar una proyección, razón por la cual se plantea una matriz con una ecuación que permite establecer un nivel de riesgo.

Se recomienda contactarse con representantes de cámaras, para lograr mayor impacto en la difusión de los resultados; además, ampliar el estudio con nuevas investigaciones; también, trabajar con otros sectores, factor que a largo plazo permita beneficiar en mayor medida a la población.

\section{Agradecimientos}

A la Asociación de Horticultores del Irazú (AHSORI) y la Asociación de Agricultores de El Guarco (AGRIRETEC), quienes hicieron posible este proyecto con la oportunidad brindada. También se agradece a la Vicerrectoría de Investigación y Extensión del Tecnológico de Costa Rica, por el apoyo para la realización de este proyecto. A la estudiante de posgrado Marcela Sierra y a los asistentes de investigación Geisel Segura, Daniel Sánchez y Dayana Picado, así como a Franciny Vega por su aporte.

\section{Referencias}

[1] K. N. Prasad, Radiation Injury Prevention and Mitigation in Humans. Florida: CRC Press, 2012.

[2] Á. Zuluaga, "Cámaras bronceadoras: mitos y realidades", Revista de la Asociación Colombiana de Dermatología y Cirugía Dermatológica, pp. 205-2010, 2009.

[3] M. González Púmariega, M. Vernhes Tamayo y Á. Sánchez Lamar, "La radiación ultravioleta. su efecto dañino y consecuencias para la salud humana", Theoría: Ciencia, Arte y Humanidades, pp. 69-80, 2009.

[4] International Food Information Service, Dictionary of Food Science and Technology, 2nd ed.. Shinfield: IFIS Publishing, 2009.

[5] F. Henao Robledo, Riesgos Físicos II., Bogotá: Ecoe Ediciones, 2008.

[6] C. Sordo y C. Gutiérrez, "Cáncer de piel y radiación solar: experiencia peruana en la prevención y detección temprana del cáncer de piel y melanoma", Revista Peruana de Medicina Experimental y Salud Pública, pp. 113-117, 2013.

[7] M. Olarte S., S. Hugo Sánchez, C. Fernando Aréchiga, R. Bañuelos V., E. Donají Ramírez y A. L. L. López L, "Daño y respuesta celular en piel por exposición prolongada a radiación UV", ANACEM, pp. 44-51, 2016.

[8] N. Pérez Consuegra y L. A. Rodríguez, "Primeras señales de recuperación de la capa de ozono", Hipótesis, Apuntes Científicos Uniandinos, pp. 14-16, 2014.

[9] CIE, "International Standard Gloval UV Index," Vienna, Austria, 2001.

[10] C. Yaipen Salazar, E. Peralta Paima, H. Rojas Cubas, A. Vivar Chao, A. Herrera Toscani y C. Díaz Vélez, "Conocimiento y actitudes de medidas de protección solar en trabajadores agrícolas", Revista del Cuerpo Médico del Hospital Nacional Almanzor Aguinaga Asenjo, pp. 207-2011, Tumán, mayo-junio 2014.

[11] V. Narváez Rosales, "Los efectos de la radiación ultravioleta y conceptos básicos de la fotoprotección", Dermatología Rev. Mex., pp. 278-283, 2001.

[12] R. Hester and R. Harrison, Causes and Environmental Implications of Increased UV-B Radiation. Cambridge: Royal Society of Chemistry, 2000.

[13] J. P. Castanedo Cázares, B. Torres Álvarez, B. Portales González, K. Martínez Rosales y D. Hernández Blanco, "Análisis de la radiación solar ultravioleta acumulada en México", Revista Medica del IMSS, pp. 26-31, 2016.

[14] M. V. Cuevas González, M. E. Vega Memije, P. Chairez Atienzo, A. G. García Calderón, J. L. González y J. C. Cuevas González, "Frecuencia de cáncer de piel en un centro de diagnóstico histopatológico en la ciudad de Durango, Durango, México", Dermatología Revista Mexicana, pp. 11-17, 2016.

[15] D. R. Sambandan and . D. Ratner, "Sunscreens: An overview and update," JAM ACAD. DERMATOL., pp. 748757, 2011.

[16] "Plan Nacional para la Prevención y Control del Cáncer 2011-2017", Ministerio de Salud, San José, 2012.

[17] R. Chang and J. W. Thoman, Physical Chemistry for the Chemical Sciences, University Science Books, 2014. 
[18] M. F. Güémez Graniel, A. Plascencia Gómez, M. J. Graniel Lavadores y K. Dzul Rosado, "Epidemiología del cáncer de piel en el Centro Dermatológico de Yucatán durante 2012", Dermatología Revista Mexicana, pp. 9-18, 2015.

[19] W. B. Grant, "An Estimate of Premature Cancer Mortality in the U.S. Due to Inadequate Doses of Solar Ultraviolet-B Radiation," American Cancer Society, pp. 1867-1874, 2002.

[20] C. Young, "Solar ultraviolet radiation and skin cancer," Occupational Medicine, pp. 82-88, 2009.

[21] K. Heinrich Bettoni, R. Pacheco Cubero, M. Solera Flores y M. Alfaro Hernández, "Series de brillo solar en Costa Rica", Ministerio de Ambiente y Energía, San José, 2013.

[22] A. L. Molina y L. Ángela , "Vitamina D y piel", Revista de la Asociación Colombiana de Dermatología y Cirugía Dermatológica, pp. 239-252, 2012.

[23] J. L. Pinedo Vega, R. Castañeda López, J. I. Dávila Rangel, F. Mireles García,, C. Ríos Martínez y A. López Saucedo, "Incidencia de cáncer de piel en Zacatecas", Revista Medica del IMSS, pp. 282-289, 2014.

[24] J. P. Castanedo Cázares, V. Lepe, A. Gordillo Moscoso y B. Moncada, "Dosis de radiación ultravioleta en escolares mexicanos", Salud Pública de México, vol. 45, n. 6, pp. 439-444, 2003.

[25] M. Ortega Resinas, F. Martín Gutiérrez y F. Camacho, "Medidas de la radiación solar ultravioleta en Sevilla", Actas Dermosifiliogr., pp. 271-275, 2001.

[26] C. E. Peters, P. A. Demers, S. Kalia, P. Hystad, P. J. Villeneuve, A.M. Nicol, N. Kreiger, and M. W. Koehoor, "Occupational exposure to solar ultraviolet radiation and the risk of prostate cancer," Occup. Environ. Med., pp. 742-748, 2016.

[27] A. M. Siani, G. R. Casale, R. Sisto, A. Colosimo, C. A. Lang y M. G. Kimlin, "Occupational Exposures to Solar Ultraviolet Radiation of Vineyard Workers in Tuscany (Italy)," Photochemistry \& Photobiology, pp. 925-934, 2011.

[28] P. Gies, K. Glanz, D. O'Riordan, T. Elliott, and E. Nehl, "Measured Occupational Solar UVR Exposures of Lifeguards in Pool Settings," American Journal of Industrial Medicine, pp. 645-653, 2009.

[29] "Índice UV solar mundial. Guía práctica". Ginebra: OMS (Organización Mundial de la Salud), 2003.

[30] H. Suárez , J. Castillo, G. Salazar , D. Acosta, C. Cadena, M. Marín , P. Utrillas y L. J. Martínez, "Variabilidad diaria y anual de radiación solar eritémica en tres regiones de la provincia de Salta", de Reunión de Trabajo de la Asociación Argentina de Energías Renovables y Medio Ambiente, Argentina, 2014.

[31] , "ICNIRP Guidelines. On Limits of exposure to ultraviolet radiation of wavelengths between $180 \mathrm{~nm}$ and 400 nm," ICNIRP (International Commission on Non-Ionizing Radiation Protection), 2004.

[32] J. M. Ríos Yuil, "Correlación entre las prácticas de exposición solar y el grado de fotodaño", Revista Médicocientífica, pp. 4-11, Panamá,mayo, 2009.

[33] M. Sierra, Exposición ocupacional a radiación ultravioleta en poblaciones agrícolas de la provincia de Cartago. Heredia, Costa Rica: Universidad Nacional, 2016.

[34] "A Guide to the UV Index," EPA, Estados Unidos, 2004. 